Toshihiro Hadano

Nagoya Math. J.

Vol. 53 (1974), 199-210

\title{
ON THE CONDUCTOR OF AN ELLIPTIC CURVE WITH A RATIONAL POINT OF ORDER 2
}

\author{
TOSHIHIRO HADANO
}

\section{Introduction}

Let $C$ be an elliptic curve (an abelian variety of dimension one) defined over the field $Q$ of rational numbers. A minimal Weierstrass model for $C$ at all primes $p$ in the sense of Néron [3] is given by a plane cubic equation of the form

$$
y^{2}+a_{1} x y+a_{3} y+x^{3}+a_{2} x^{2}+a_{4} x+a_{6}=0,
$$

where $a_{j}$ belongs to the ring $Z$ of integers of $Q$, the zero of $C$ being the point of infinity.

Following Weil, we define the conductor $N$ of $C$ by

$$
N=\prod_{\text {a.l } p} p^{\left(\operatorname{ord}_{p} \Delta+1-n_{p}\right)}
$$

where $\Delta$ denotes the discriminant of $C$, and $n_{p}$ is the number of components of the Néron reduction of $C$ over $Q$ without counting multiplicities. It is well-known that the $p$-exponent of $N$ is

$$
\operatorname{ord}_{p} \Delta+1-n_{p}= \begin{cases}0 & \text { for non-degenerate reduction } \\ 1 & \text { for multiplicative reduction } \\ 2 & \text { for additive reduction and } p \neq 2,3 \\ \geqq 2 & \text { for additive reduction and } p=2,3\end{cases}
$$

Therefore both $N$ and $\Delta$ of a minimal model are divisible exactly by those primes at which $C$ has degenerate reduction. (See Ogg [6], [7]).

We consider the problem to find all the elliptic curves over $\boldsymbol{Q}$ of given conductor $N$. As we may reduce this problem to find the rational solutions of the diophantine equation $y^{2}=x^{3}+k$ with $k \in Z$, there are only finitely many such curves by virtue of Thue's theorem. Ogg [5], [6] has found all the curves by showing that they have a rational point

Received September 27, 1973. 
of order 2 for $N=2^{m}, 3 \cdot 2^{m} 9 \cdot 2^{m}$, while Vélu [8] found all the curves of $N=11^{m}$ under the Weil's conjecture for $\Gamma_{0}(N)$. On the other hand, Miyawaki [1] has culculated all the curves of prime power conductor with a rational point of finite order $>2$.

In this paper we treat the curves of $N=p^{m}$ and $2^{m} p^{n}$ (for this case, see [10] as résumé) with a rational point of order 2 . For $N=p^{m}$, we can find all admissible $p$, and, a fortiori, all the curves for each $p$. (Section 3). For $N=2^{m} p^{n}$, we can find all the curves under an assumption which can be eliminated for 'non-large' $p$ with $p \equiv 3$ or $5(\bmod 8)$. Moreover, we get some results on the elliptic curve which has multiplicative reduction at 2 and $p$, and these are generalizations of the results of Ogg [6]. (Section 4). In Appendix all the elliptic curves of 3-power conductor are determined.

\section{Diophantine lemma}

We prepare all the diophantine results we need afterwards.

LEMMA. The only non-zero integral solutions of the equations below for a given odd prime $p$ are as follows:

1) If $X^{2}-1=2^{\alpha} p^{\beta}$, then $\left(|X|, 2^{\alpha} p^{\beta}\right)=(2,3),\left(3,2^{3}\right),\left(5,2^{33}\right),\left(7,2^{4} 3\right),\left(9,2^{4} 5\right)$, $\left(17,2^{5} 3^{2}\right)$ for $p \equiv 3$ or $5(\bmod 8)$, and $\beta=1, p=2^{\alpha-2} \pm 1(\alpha \geqq 5)$ for $p \equiv 1$ or $7(\bmod 8)$.

2) If $X^{2}+1=2^{\alpha} p^{\beta}$, then $\left(|X|, 2^{\alpha} p^{\beta}\right)=(1,2)$ for $p \equiv 3(\bmod 4)$, and either $\alpha=0, \beta=1$ or $\alpha=1, \beta=1,2,4$ for $p \equiv 1(\bmod 4)$. In particular we have $\beta=4$ if and only if $p=13,|X|=239$.

3) If $2 X^{2}-1=p^{\alpha}, \alpha>0$, then there is no solution for $p \equiv 3$ or $5(\bmod 8)$.

4) If $2 X^{2}+1=p^{\alpha}, \alpha>0$, then $\alpha=1,2$ or $\left(|X|, p^{\alpha}\right)=\left(11,3^{5}\right)$ for $p \equiv 1$ or $3(\bmod 8)$, and there is no solution for $p \equiv 5$ or $7(\bmod 8)$.

5) We assume here that $p$ satisfies the conjecture of Ankeny-ArtinChowla and the analogy (See [2], Chapter 8) for $p \equiv 3$ or $5(\bmod 8)$. If $\left| \pm p^{\alpha}-X^{2}\right|=2^{\beta}$, then $\left( \pm p^{\alpha},|X|\right)=(1,3),(-1,1),(3,2),(-3,1),\left(3^{2}, 1\right),\left(3^{2}, 5\right)$, $\left(3^{3}, 5\right),\left(3^{4}, 7\right)$ or $\alpha=\beta=1$ for $p \equiv 3(\bmod 8)$, and $\left( \pm p^{\alpha},|X|\right)=(1,3),(-1,1)$, $\left(5^{2}, 3\right),\left(5^{3}, 11\right), \alpha=1, \beta=0$ or $\alpha=1, \beta=2$ for $p \equiv 5(\bmod 8)$.

6) If $p X^{2}-Y= \pm 2^{\alpha}$, and $Y= \pm 2^{\beta}$, then either $2|X, 4| Y$, or $(|X|, Y)$ $=(1,4),(1,2),(1,1),(1,-1)$ for $p=3,(1,4),(1,1)$ for $p=5$, and there is no solution for $p \neq 3,5$.

7) If $X^{2}-64=p^{\alpha}$, then $\left(|X|, p^{\alpha}\right)=(9,17)$.

8) If $X^{2}+64=p^{\alpha}$, then $\left(|X|, p^{\alpha}\right)=\left(15,17^{2}\right)$ or $\alpha=1$ for all $p$. 
This lemma except 7) and 8) is a generalization of Diophantine lemma of Ogg [6]. The methods for solving these equations are standard and elementary. We refer to each parts of this lemma as $D_{1}, \ldots, D_{8}$. $D_{1}$ is easy by [2], Chapter 30. $D_{2}$ may be solved by factorization in $Z[\sqrt{-1}]$ and Ljunggren's result in [2], Chapter 28. $D_{3}$ is easy. $D_{4}$ and $D_{5}$ may be solved by the congruence method and the results of Pell's equation. $D_{6}$ and $D_{7}$ are easy. $D_{8}$ may be solved by factorization in $Z[\sqrt{-1}]$.

\section{The case of $N=p^{m}$}

Let $C$ be an elliptic curve of conductor $N=p^{m}$ with a rational point of order 2. Then we have $m=1$ or 2 from Section 1 if $p \neq 2,3$ (cf. Appendix) and we have a defining equation for $C$ of the form

$$
y^{2}+x^{3}+a_{2} x^{2}+a_{4} x=0
$$

with $a_{j} \in Z$, minimal at all $p \neq 2$, and such that we do not have $2^{2} \mid a_{2}$ and $2^{4} \mid a_{4}$. This curve is isomorphic to

$$
y^{2}+x y+x^{3}+\left(\frac{a_{2}+1}{4}\right) x^{2}+\frac{a_{4}}{16} x=0,
$$

minimal at all $p$. If these coefficients are not integers, they can be made integers by a translation.

Now we propose to find all possible $p$ such that the discriminant of (3.2) is

$$
\Delta=2^{-8} a_{4}^{2}\left(a_{2}^{2}-4 a_{4}\right)= \pm p^{2} .
$$

This result will give the determination of all $C$ above up to isomorphisms. At first, dividing the curve (3.2) by the group generated by $(x, y)=(0,0)$, we have an isogenous curve of degree 2 given by

$$
y^{2}+x y+x^{3}+\left(\frac{1-2 a_{2}}{4}\right) x^{2}+\left(\frac{a_{2}^{2}-4 a_{4}}{16}\right) x=0,
$$

which also has a rational point $(x, y)=(0,0)$ of order 2 . Its discriminant is $2^{-4} a_{4}\left(a_{2}^{2}-4 a_{4}\right)^{2}= \pm p^{u} 2^{12 k} \quad(k \in Z, k \geqq 0)$, since (3.3) is not necessarily minimal at $p=2$ and there is a relation, in general, $12 \mid\left(\operatorname{ord}_{p} \Delta^{\prime}-\operatorname{ord}_{p} \Delta\right)$ between the discriminant $\Delta^{\prime}$ of a non-minimal model and the discriminant $\Delta$ of its minimal model. Hence we have $p^{2 \lambda}= \pm 2^{12 k-12} a_{4}^{3} p^{u}$, and so $\left|a_{4}\right|=1$, $16, p^{\alpha}$ or $16 p^{\alpha}$ and $k=0$ or 1 . On the other hand, either $a_{2}$ is odd or 
$2 \| a_{2}$ as we see below, and we see that only if $a_{2} \equiv 3(\bmod 4)$ or $a_{2} \equiv 2$ (mod 8 ) according as $2 \nmid a_{2}$ or $2 \| a_{2}$ respectively, we may rewrite the equation (3.3) to the minimal equation of integral coefficients by a suitable translation.

If $a_{4}= \pm 1$, then $a_{2}=2 b_{2}$ is even, so $\left|b_{2}^{2} \pm 1\right|=2^{6} p^{2}$, and by $D_{1}$ and $D_{2}$ we get $\left(p, a_{2}, a_{4}\right)=(17,66,1)$. If $a_{4}= \pm 16$, then $\left|a_{2}^{2} \pm 64\right|=p^{\lambda}$; by $D_{7}$ and $D_{8}$ we get $\left(p, a_{2}, a_{4}\right)=(17,-9,16),(17,15,-16)$, or $\left(X^{2}+64, X,-16\right)$. If $a_{4}= \pm p^{\alpha}$, then $a_{2}=2 b_{2}$ is even, so $\left|b_{2}^{2} \pm p^{\alpha}\right|=2^{6} p^{\lambda-2 \alpha}$. Suppose first $\lambda=2 \alpha$, then $\left|b_{2}^{2} \pm 64\right|=p^{\alpha} ;$ by $D_{7}$ and $D_{8}$ we get $\left(p, a_{2}, a_{4}\right)=(17,18,17)$, $\left(17,-30,17^{2}\right)$ or $\left(X^{2}+64,-2 X, X^{2}+64\right)$. Henceforth put $b_{2}=p^{t} c_{2}\left(p \nmid c_{2}\right)$ so that $\left|p^{2 t} c_{2}^{2} \pm p^{\alpha}\right|=2^{6} p^{\lambda-2 \alpha}$. If $\alpha \geqq 4$, then $t=1$ since otherwise we can find a better model, so $c_{2}^{2} \pm p^{\alpha-2}= \pm 2^{6} p^{\lambda-2 \alpha-2}$ and by $D_{7}$ and $D_{8}$ we get $\left(p, a_{2}, a_{4}\right)=\left(17,-510,17^{4}\right)$. If $\alpha=3$, then $\left|c_{2}^{2} \pm p^{3-2 t}\right|=2^{6} p^{\lambda-2 t-6}$ or $\left|p^{2 t-3} c_{2}^{2} \pm 1\right|=2^{6} p^{\lambda-9}$, and by $D_{7}$ and $D_{8}$ we get $\left(p, a_{2}, a_{4}\right)=\left(17,306,17^{3}\right)$, $\left(X^{2}+64,2 X\left(X^{2}+64\right),\left(X^{2}+64\right)^{3}\right)$ or $\left(7,-294,-7^{3}\right)$. If $\alpha=2$, then $\left|c_{2}^{2} \pm p^{2-2 t}\right|=2^{6} p^{\lambda-2 t-4}$ or $\left|p^{2 t-2} c_{2}^{2} \pm 1\right|=2^{6} p^{\lambda-6}$, and by $D_{1}, D_{2}, D_{7}$ and $D_{8}$ we get $\left(p, a_{2}, a_{4}\right)=\left(17,66 \cdot 17,17^{2}\right)$. If $\alpha=1$, then $\left|p^{2 t-1} c_{2}^{2} \pm 1\right|=2^{6} p^{\lambda-3}$ and we get $\left(p, a_{2}, a_{4}\right)=(7,42,-7)$. Lastly if $a_{4}= \pm 16 p^{\alpha}$, then $\left|a_{2}^{2} \pm 2^{6} p^{\alpha}\right|=$ $p^{\lambda-2 \alpha}$. Therefore similarly to above, we get $\left(p, a_{2}, a_{4}\right)=(17,-33,16 \cdot 17)$, $\left(17,-17 \cdot 9,16 \cdot 17^{2}\right),\left(17,17 \cdot 15,-16 \cdot 17^{2}\right),\left(17,17 \cdot 33,16 \cdot 17^{3}\right),\left(7,147,16 \cdot 7^{3}\right)$, $\left(X^{2}+64, X\left(X^{2}+64\right),-16\left(X^{2}+64\right)^{2}\right)$ or $(7,-21,16 \cdot 7)$. This completes all cases.

By identifying the isomorphic curves each other we have

THEOREM I. There are elliptic curves of conductor $N=p^{m}$, (where $p \neq 2$ and $m=1$ or 2 ), with a rational point of order 2 for $p=7,17$ and primes $p$ such that $p-64$ is square.

The minimal models with integral coefficients for $p=7,17,73$ are following :

Table 1.

\begin{tabular}{c|l|r|l}
\hline$N$ & minimal equation & \multicolumn{1}{c|}{$\Delta$} & \multicolumn{1}{c}{ 2-division points $(x, y) \neq \infty$} \\
\hline $7^{2}$ & $y^{2}+x y+x^{3}-5 x^{2}+7 x=0$ & $-7^{3}$ & $(0,0)$ \\
& $y^{2}+x y+x^{3}-5 x^{2}-28 x+3 \cdot 7^{2}=0$ & $7^{3}$ & $\left(\frac{21}{4},-\frac{21}{8}\right)$ \\
& $y^{2}+x y+x^{3}+37 x^{2}+7^{3} x=0$ & $-7^{9}$ & $(0,0)$ \\
& $y^{2}+x y+x^{3}+37 x^{2}-4 \cdot 7^{3} x-3 \cdot 7^{5}=0$ & $7^{9}$ & $\left(-\frac{147}{4}, \frac{147}{8}\right)$
\end{tabular}




\begin{tabular}{|c|c|c|c|}
\hline$N$ & minimal equation & $\Delta$ & 2-division points $(x, y) \neq \infty$ \\
\hline \multirow[t]{4}{*}{17} & $y^{2}+x y+x^{3}-2 x^{2}+x=0$ & 17 & $(0,0),(1,0),(1,-1)$ \\
\hline & $y^{2}+x y+x^{3}+16 x^{2}-8 x+1=0$ & 17 & $\left(\frac{1}{4},-\frac{1}{8}\right)$ \\
\hline & $y^{2}+x y+x^{3}+4 x^{2}-x=0$ & $17^{2}$ & $(0,0),(-4,2),\left(\frac{1}{4},-\frac{1}{8}\right)$ \\
\hline & $y^{2}+x y+x^{3}-20 x^{2}+136 x-17^{2}=0$ & $-17^{4}$ & $\left(\frac{17}{4},-\frac{17}{8}\right),(0, \pm 17)$ \\
\hline \multirow[t]{4}{*}{$17^{2}$} & $y^{2}+x y+x^{3}-38 x^{2}+17^{2} x=0$ & $17^{7}$ & $\begin{array}{l}(0,0),(-17,9 \cdot 17) \\
(-17,-8 \cdot 17)\end{array}$ \\
\hline & $y^{2}+x y+x^{3}+268 x^{2}-8 \cdot 17^{2} x+17^{3}=0$ & $17^{7}$ & $\left(\frac{17}{4},-\frac{17}{8}\right)$ \\
\hline & $y^{2}+x y+x^{3}-140 x^{2}+17^{3} x=0$ & $17^{8}$ & $(0,0),(68,-34),\left(\frac{17^{2}}{4},-\frac{17^{2}}{8}\right)$ \\
\hline & $y^{2}+x y+x^{3}-344 x^{2}+8 \cdot 17^{3} x-17^{5}=0$ & $-17^{10}$ & $\left(\frac{17^{2}}{4},-\frac{17^{2}}{8}\right)$ \\
\hline \multirow[t]{2}{*}{73} & $y^{2}+x y+x^{3}+x^{2}-x=0$ & 73 & $(0,0)$ \\
\hline & $y^{2}+x y+x^{3}+x^{2}+4 x+3=0$ & $-73^{2}$ & $\left(-\frac{3}{4}, \frac{3}{8}\right)$ \\
\hline \multirow[t]{2}{*}{$73^{2}$} & $y^{2}+x y+x^{3}+55 x^{2}-73^{2} x=0$ & $73^{7}$ & $(0,0)$ \\
\hline & $y^{2}+x y+x^{3}+55 x^{2}+4 \cdot 73^{2} x+3 \cdot 73^{3}=0$ & $-73^{8}$ & $\left(-\frac{219}{4}, \frac{219}{8}\right)$ \\
\hline
\end{tabular}

Remark. We see that the members in each $N$ above are isogenous to each other. (See Vélu [9]). For $p=2$, see Ogg [5]. It is well known that $N \neq 7$.

\section{The case of $N=2^{m} p^{n}$}

In this section we deal with the case $N=2^{m} p^{n}$ for odd prime $p$ and generalize the results of Ogg using his ideas ([6], §2).

Let $K=Q\left(C_{2}\right)$ be a Galois field generated by the group $C_{2}$ of 2-division points on the elliptic curve $C$ defind over $Q$. For each prime $p$, $e_{p}$ denotes the ramification degree of $K / \boldsymbol{Q}$ at $p$. Then we know the following results :

LEMMA (Ogg [6]). (1) If $C$ has non-degenerate reduction at each $p \neq 2$, then $e_{p}=1$.

(2) If $C$ has multiplicative reduction at all $p$, then $e_{p}=1$ or 2 .

(3) Suppose $C$ has no non-zero point of order 2 in rational coordinates, then $K / k$ is cyclic of degree 3 over a field $k$ of degree 1 or 2 over $Q$. Suppose furthermore $e_{p}=1$ or 2 for all $p$. Then the class number of 
$k$ is divisible by 3.

Now let $p$ be an odd prime such that none of the class numbers of four fields $Q(\sqrt{ \pm p}), Q(\sqrt{ \pm 2 p})$ is divisible by 3 , and fix this $p$. Suppose $C$ has non-degenerate reduction (i.e. good reduction) at all primes $q \neq 2$, $p$. Then $e_{q}=1$ by (1) in Lemma, and if the first conditions of (3) in Lemma is satisfied, then $k$ in (3) is $Q, Q(\sqrt{-1}), Q(\sqrt{ \pm 2}), Q(\sqrt{ \pm p})$ or $Q(\sqrt{ \pm 2 p})$. Hence $3 \mid e_{2}$ or $3 \mid e_{p}$. Therefore $3 \mid e_{2}$ by virtue of (2) in Lemma if $N=2^{m} p$, that is, $C$ has a rational point of order 2 if $e_{2}=1$ or 2 and $N=2^{m} p$. In particular by (2) in Lemma $C$ has a rational point of order 2 if $N=2 p$. So we can generalize Ogg's result:

THEOREM II. If none of the class numbers of four quadratic fields $Q(\sqrt{ \pm p}), Q(\sqrt{ \pm 2 p})$ for a prime $p \equiv 3$ or $5(\bmod 8)$ is divisible by 3 , then there are no elliptic curves of conductor $N=2 p$.

Proof. If there exists such a curve, we can choose an equation

$$
y^{2}+x^{3}+a_{2} x^{2}+a_{4} x=0
$$

with $a_{j} \in Z$, minimal at all $p \neq 2$. We also assume that we do not have $2^{2} \mid a_{2}$ and $2^{4} \mid a_{4}$. Since we have multiplicative reduction at 2 and $p$, $\operatorname{ord}_{2} j<0$ and $p \nmid a_{2}$ (cf. [3]), where $j=2^{12}\left(a_{2}^{2}-3 a_{4}\right)^{3} \Delta^{-1}$ is the invariant of the curve with the discriminant $\Delta=2^{4} a_{4}^{2}\left(a_{2}^{2}-4 a_{4}\right)= \pm 2^{\mu} p^{\nu}$. Hence we have $\mu=\operatorname{ord}_{2} \Delta>12$. If $a_{2}$ is odd, then $a_{2}^{2}-4 a_{4}= \pm p^{\alpha}, \operatorname{ord}_{2}\left(4 a_{4}\right)>6$. If $p \mid a_{4}$, then $a_{2}^{2} \pm 1=4 a_{4}=2^{\alpha} p^{\beta}$, which is impossible by $D_{1}$ and $D_{2}$ since $\alpha>6$. If $p \nmid a_{4}$, then $\left| \pm p^{\alpha}-a_{2}^{2}\right|=\left|4 a_{4}\right|=2^{\beta}, \beta>6$, which is also impossible by $D_{5}$ (without the assumption there). Then we see that this theorem can be proven by the same method as used by Ogg to show $N \neq 10,12$ in [6], $\S 4$. (Replace Diophantine lemma there with our $D_{1}$ and $D_{5}$ ! 'Of type $C 1$ ' in his proof should be 'of type $C 2$ '.)

For example, we have $p=37,43,67,197,227$ etc. except $p=3,5,11$. However, it is well-known that this is not true for $p \equiv 1$ or $7(\bmod 8)$, but on the other hand we have

THEOREM III. If none of the class numbers of four quadratic fields $Q(\sqrt{ \pm p}), Q(\sqrt{ \pm 2 p})$ for a prime $p \equiv 1$ or $7(\bmod 8)$ is divisible by 3 , then the elliptic curves of conductor $N=2^{m} p,(m>0)$, have a rational point of order 2. 
Proof. As a defining equation for a curve $C$ of $N=2^{m} p$, we can take

$$
y^{2}+x^{3}+a_{2} x^{2}+a_{4} x+a_{6}=0
$$

with $a_{j} \in Z$, minimal at all $p \neq 2$. If $3 \nmid a_{2}$, then we get an equation

$$
y^{2}+x^{3}+a_{4} x+a_{6}=0
$$

with $a_{j} \in Z$, minimal at all $p \neq 2,3$ and such that we do not have $2^{4} \mid a_{4}$ and $2^{6} \mid a_{6}$. The discriminant $\Delta$ of this curve is

$$
\Delta=-2^{4}\left(4 a_{4}^{3}+27 a_{6}^{2}\right)= \pm 2^{\mu} 3^{12} p^{\nu}, \quad(\mu, \nu>0) .
$$

Suppose that $C$ has no rational point of order 2 , then an irrational point $(x, y)$ of order 2 is $(r, 0)$, where $r$ is a root of $f(X)=X^{3}+a_{4} X+a_{6}$ and $r \notin Q$. Therefore the ramification degree $e_{2}$ at the prime 2 of $Q(r) / Q$ is 3 under the assumption as we have seen. Considering the discriminant of this cubic field, we see that $a_{6}$ is even. If $a_{4}$ is odd, then $x=0$ refines to a root $r$ of $f(X)$ in $Q_{2}$ by Newton's method. This is a contradiction. Put $a_{4}=-2 u, a_{6}=2 v$. Then $8 u^{3}-27 v^{2}= \pm 2^{\mu-6} 3^{12} p^{\nu}$, so $v$ is even, since otherwise $8 u^{3}-27 v^{2}= \pm 3^{12} p^{\nu}$, which is impossible modulo 8 for $p \equiv 1$ or $7(\bmod 8)$. Put $v=2 v_{1}$. Then we have $f(X)=X^{3}-2 u X$ $+2^{2} v_{1}$, hence $u$ is even by $e_{2}=3$. Put $u=2 u_{1}$, then $16 u_{1}^{3}-27 v_{1}^{2}=$ $\pm 2^{\mu-8} 3^{12} p^{\nu}$, so $v_{1}$ is even, since otherwise $16 u_{1}^{3}-27 v_{1}^{2}= \pm 3^{12} p^{\nu}$, which is impossible as above. Therefore we have $2^{2} \mid a_{4}$ and $2^{3} \mid a_{6}$. Thus to solve $f(X)=0$ is the same thing as to solve

$$
2^{-3} f(2 X)=X^{3}+2^{-2} a_{4} X+2^{-3} a_{6} .
$$

Hence repeating the above arguments, we have $2^{4} \mid a_{4}$ and $2^{6} \mid a_{6}$, and this is a contradiction. If $3 \mid a_{2}$, then we get (4.1) with $a_{j} \in Z$, minimal at all $p \neq 2$, such that the discriminant

$$
\Delta=-2^{4}\left(4 a_{4}^{3}+27 a_{6}^{2}\right)= \pm 2^{\mu} p^{\nu} \quad(\mu, \nu>0)
$$

and such that we do not have $2^{4} \mid a_{4}$ and $2^{6} \mid a_{6}$. In the same manner as above, we can complete the proof of this case, too.

For example we have $p=7,17,41,47,73,97$ etc. as such $p$.

In another direction:

THEOREM IV. All the elliptic curves of the conductor $N=2^{m} p^{n}$, where $p \equiv 3$ or $5(\bmod 8)$ and $p \neq 3$, that have a rational point of order 
2 are effectively determined under the conjecture of Ankeny-Artin-Chowla and the analogy. In particular if $p-2$ or $p-4$ is a square number, then the assumption on the conjecture can be eliminated.

Proof. We can take a defining equation for $C$ of the form

$$
y^{2}+x^{3}+a_{2} x^{2}+a_{4} x=0
$$

with $a_{j} \in Z$, minimal at all $p \neq 2$, and such that we do not have $2^{2} \mid a_{2}$ and $2^{4} \mid a_{4}$. The discriminant of this model is

$$
\Delta=2^{4} a_{4}^{2}\left(a_{2}^{2}-4 a_{4}\right)= \pm 2^{\mu} p^{\nu} .
$$

It is sufficient to find all the pairs $\left(a_{2}, a_{4}\right)$ satisfying (4.3) for a given $p$. Noting that $p \nmid a_{2}$ (resp. $p \mid a_{2}$ ) if $N=2^{m} p$ (resp. $N=2^{m} p^{2}$ ), we can get all the pairs $\left(a_{2}, a_{4}\right)$, up to isomorphisms, by virtue of Diophantine lemma $D_{1}, \cdots, D_{6}$ in view of the fact that $2^{2} \nmid a_{2}$ and $2^{4} \nmid a_{4}$. (For details, see Ogg [6], § 3.)

Remark. We know that $n=1$ or 2 only if $p \geqq 5$. For $p=3$, Ogg [6] has found all the curves of conductor $N=3 \cdot 2^{m}$ and $9 \cdot 2^{m}$ by showing that they have a rational point of order 2 (cf. [4]), and Coghlan has found in his thesis all the curves of conductor $N=2^{m} 3^{n}$. For example, if $N=2^{m} 5$ in our case, then $2 \leqq m \leqq 7$ and there are 56 curves with a rational point of order 2 . We can prove, in general, that the integer $m$ is not larger than 8. Moreover, we see that the equation (4.2) is minimal at all $p$ (including $p=2$ ), in fact, otherwise we can consider the same situation as in Section 3 for $N=2^{m} p^{n}$ to show that we cannot find the pairs $\left(a_{2}, a_{4}\right)$ of the equation (3.2) since the equation $\left|X^{2} \pm p^{\alpha}\right|$ $=2^{\beta}, \beta>6$, has no integer solutions for $p \equiv 3$ or $5(\bmod 8)$ by $D_{5}$. For

$p \equiv 1$ or $7(\bmod 8)$, it seems to be difficult to solve the equations of $D_{3}$, $D_{5}$ (especially $D_{5}$ ) in general, but the theorem remains valid for all $p>3$ so long as those equations are solved.

\section{Supplementary discussions}

We can find all the curves of some other conductors $N$ with a rational point of order 2 so long as the corresponding diophantine equations can be solved as in the previous sections. In fact, for example, we can find all the curves of conductor $N=p^{m} q^{n}$, where $m, n>0, p$ and $q$ are primes such that $p \equiv 3, q \equiv 5(\bmod 8)$, with a rational point 
of order 2. By solving the equations

$$
\begin{gathered}
X^{2} \pm 64= \pm p^{\alpha} q^{\beta}, \quad X^{2} \pm 1=2^{6} p^{\alpha} q^{\beta}, \quad\left|p^{\alpha} X^{2} \pm 1\right|=2^{6} q^{\beta}, \\
\left|p^{\alpha} \pm 2^{6} q^{\beta}\right|=X^{2}, \quad\left|p^{\alpha} X^{2} \pm 64\right|=q^{\beta}
\end{gathered}
$$

induced from the defined equation (3.2) in Section 3 with $\Delta= \pm p^{\mu} q^{\nu}$, we get 136 curves of $(p, q)=(3,5),(3,13),(11,5),(19,5),(3,37),(3,61)$, $(59,5),(11,53)$ and $m, n=1$ or 2 . Moreover, a fortiori, we can find all the curves of a given conductor $N$ with a rational point of order $r>2$ so long as the corresponding diophantine equations can be solved. In fact, for example, if $N=2^{m} p^{n}$, and $r=4$ (cyclic), then such curves can be defined by

$$
y^{2}+x^{3} \pm\left(s^{2}+2 t\right) x^{2}+t^{2} x=0
$$

with $s, t \in Z, s>0$, minimal at all $p \neq 2$, and these curves are isogenous to

$$
y^{2}+x\left(x \mp s^{2}\right)\left(x \mp s^{2} \mp 4 t\right)=0,
$$

which have three rational points of order 2 . Then we have either $p=$ $2^{k} \pm 1(k \geqq 1)$ or $N=2^{5} p^{2}, 2^{6} p^{2}$ for all $p$. In particular, we have only $N=17^{n}$ for $m=0$ and the curves are included in Table 1 in section 3 . As another example, suppose $N=2^{m} p^{n}$ and $r=6$ (non-cyclic, that is, curves which have both a rational point of order 2 and of order 3); then we have $N=14,20,34$ and 36 as Table 2 below. Note that there exist two curves; $y^{2}+x y+x^{3}-45 x^{2}+2^{9} x=0, y^{2}+x y+x^{3}-45 x^{2}-$ $2^{11} x+2^{9} 181=0$ (resp. $y^{2}+x^{3}+11 x^{2}-x=0, y^{2}+x^{3}-22 x^{2}+125 x=0$; $\left.y^{2}+x^{3}-9 x^{2}+27 x=0, y^{2}+x^{3}+18 x^{2}-27 x=0\right)$ in addition to these for $N=14$ (resp. $20 ; 36$ ), and the 6 or 4 curves for $N=14,20,36$ are isogenous to each other of degree 2 or 3.

Table 2.

\begin{tabular}{l|r|l|r|r|c}
\hline$N$ & \multicolumn{1}{|c|}{ minimal model } & \multicolumn{1}{c|}{$\Delta$} & $j$ & 2-isogenous to: \\
\hline 14 & 1 & $y^{2}+x y+y+x^{3}=0$ & $-2^{2} 7$ & $-5^{6} 2^{-2} 7^{-1}$ & $2, *$ \\
14 & 2 & $y^{2}-5 x y+y+x^{3}=0$ & $2 \cdot 7^{2}$ & $5^{3} 101^{3} 2^{-1} 7^{-2}$ & $1, * *$ \\
14 & 3 & $y^{2}-5 x y+7 y+x^{3}=0$ & $-2^{6} 7^{3}$ & $5^{3} 43^{3} 2^{-6} 7^{-3}$ & $4, *$ \\
14 & 4 & $y^{2}-11 x y+49 y+x^{3}=0$ & $2^{3} 7^{6}$ & $5^{3} 11^{3} 31^{3} 2^{-3} 7^{-6}$ & $3, * *$ \\
20 & 5 & $y^{2}+x^{3}-x^{2}-x=0$ & $2^{4} 5$ & $2^{14} 5^{-1}$ & 6 \\
20 & 6 & $y^{2}+x^{3}+2 x^{2}+5 x=0$ & $-2^{8} 5^{2}$ & $2^{4} 11^{3} 5^{-2}$ & 5 \\
34 & 7 & $y^{2}+x y+x^{3}+6 x^{2}+8 x=0$ & $2^{6} 17$ & $5^{3} 29^{3} 2^{-6} 17^{-1}$ & 8
\end{tabular}




\begin{tabular}{l|r|l|c|c|c}
\hline \multicolumn{1}{c|}{} & \multicolumn{1}{|c|}{ minimal model } & $\Delta$ & $j$ & 2-isogenous to: \\
\hline 34 & 8 & $y^{2}+x y+x^{3}-43 x-105=0$ & $2^{3} 17^{2}$ & $5^{3} 7^{3} 59^{3} 2^{-3} 17^{-2}$ & 7 \\
36 & 9 & $y^{2}+x^{3}+3 x^{2}+3 x=0$ & $-2^{4} 3^{3}$ & 0 & 10 \\
36 & 10 & $y^{2}+x^{3}-6 x^{2}-3 x=0$ & $2^{8} 3^{3}$ & $2^{4} 3^{3} 5^{3}$ & 9 \\
\hline
\end{tabular}

* (resp. **) denotes the isogeny of degree 3 between the curves with the same symbol.

\section{APPENDIX}

We can find all the elliptic curves of 3-power conductor defined over $Q$, up to isomorphism, as listed in Table below. Coghlan found all the curves of $N=2^{m} 3^{n}$ in his thesis, in which the curves of $N=3^{n}$ are dealt with in a manner different from below.

The minimal model (1.1) in Section 1 with $\Delta= \pm 3^{\mu}$ is reduced to

$$
y^{2}+x^{3}+a_{4} x+a_{6}=0
$$

with $a_{j} \in Z$, minimal at all $p \neq 2,3$, and with the discriminant

$$
-2^{4}\left(4 a_{4}^{3}+27 a_{6}^{2}\right)= \pm 2^{12} 3^{\nu} .
$$

This may be reduced to the diophantine equation

$$
y^{2}=x^{3} \pm 3^{\nu-3} \quad \text { with } \quad a_{4}=-2^{23} 3, a_{6}=2^{4} y,
$$

where $\nu \geqq 3$ and $x, y \in Z$. In fact, it is well-known that there are no elliptic curves of the conductor $N=3^{n}$ with $0 \leqq n \leqq 2$. In order to show that $x, y \in Z$, we have to show that the equations $y^{2}=x^{3} \pm 2^{6} 3^{\nu-3}$ have no odd integral solutions. Since the ranks of the Mordell-Weil groups of the elliptic curves $y^{2}=x^{3} \pm 1, y^{2}=x^{3}-3$ and $y^{2}=x^{3}-9$ are all zero, it is sufficient to show that $y^{2}=x^{3}+k$ for $k=2^{6} 3^{2}, 2^{6} 3,-2^{6} 3^{4}$, and $-2^{6} 3^{5}$ has only integral solutions. This is easily done in a familiar manner.

LEMMA. The elliptic curve with the conductor $N=3^{m}$ is of the form

$$
y^{2}+y+x^{3}+a_{4} x+a_{6}=0
$$

with $a_{j} \in \boldsymbol{Z}$, minimal at all $p$.

Proof. By a transformation the equation (1.1) is reduced to 


$$
y^{2}+x^{3}+\left(4 a_{2}-a_{1}^{2}\right) x^{2}+8\left(2 a_{4}-a_{1} a_{3}\right) x+16\left(4 a_{6}-a_{3}^{2}\right)=0
$$

or

$$
\begin{aligned}
y^{2}+x^{3} & +3^{4}\left\{24\left(2 a_{4}-a_{1} a_{3}\right)-\left(4 a_{2}-a_{1}^{2}\right)^{2}\right\} x \\
& +3^{3}\left\{2\left(4 a_{2}-a_{1}^{2}\right)^{3}+16 \cdot 3^{3}\left(4 a_{6}-a_{3}^{2}\right)-8 \cdot 3^{2}\left(4 a_{2}-a_{1}^{2}\right)\left(2 a_{4}-a_{1} a_{3}\right)\right\}=0
\end{aligned}
$$

with the discriminant $\pm 2^{12} 3^{\mu}$ or $\pm 2^{12} 3^{\mu+12}$ respectively. Then, since this should coincide with (A-1), $a_{1}$ is even by (A-2). If $a_{3}$ is even, then (A-2) is minimal at 2 , and so the conductor of the model is $2^{m} 3^{n}(m>0)$. Hence we may put $a_{3}=1$ by a transformation $x \rightarrow x+r(r \in Z)$. Finally $3 \mid a_{2}$ in (1.1) since $C$ has an additive reduction at 3 . Hence we may put $a_{2}=0$.

Now we can determine all the curves of $N=3^{n}$. By the above Lemma, the discriminant is

$$
\Delta=-2^{6} a_{4}^{3}-27\left(1-4 a_{6}\right)^{2}= \pm 3^{\mu},
$$

and so $\left(1-4 a_{6}\right)^{2}=\left(4 c_{4}\right)^{3}+3^{\mu-3}$ with $a_{4}=-3 c_{4}$. We see that $\nu$ is odd, looking modulo 8. On the other hand, all the integral solutions of the equation $y^{2}=x^{3}+3^{n}$ with $x \equiv 0(\bmod 4), 2 \mid n$ and $n \leqq 10$ are given by:

\begin{tabular}{c|c|c|c|c|c|c}
\hline$n$ & 0 & 2 & 4 & 6 & 8 & 10 \\
\hline $\begin{array}{c}\text { solutions } \\
(x,|y|)\end{array}$ & $(0,1)$ & $\begin{array}{c}(0,3) \\
(40,253)\end{array}$ & $\left(0,3^{2}\right)$ & $\left(0,3^{3}\right)$ & $\begin{array}{c}\left(0,3^{4}\right) \\
\left(40 \cdot 3^{2}, 253 \cdot 3^{3}\right)\end{array}$ & $\left(0,3^{5}\right)$ \\
\hline
\end{tabular}

Therefore we get the Table below by taking into consideration that we have a better model whenever $\mu \geqq 15$. The 8 curves listed are all nonisomorphic and the 4 curves of $N=27$ are isogenous to each other of degree 3.

Table: Curves of conductor $N=3^{2}$ and of the form $y^{2}+y+x^{3}+$ $a_{4} x+a_{6}=0$

\begin{tabular}{c|r|r|c|c|c|r|c|c|c}
\hline Curve & \multicolumn{1}{|c|}{$a_{4}$} & \multicolumn{1}{|c|}{$a_{6}$} & $\Delta$ & $N$ & 3 -type & $j$ & $C_{\boldsymbol{Q}, 3} \neq 0 ?$ & $\begin{array}{l}\text { 3-isogenous } \\
\text { to: }\end{array}$ & $\begin{array}{c}\text { isomorphic } \\
/ \boldsymbol{Q}(\sqrt{-3}) \text { to: }\end{array}$ \\
\hline 1 & 0 & 0 & $-3^{3}$ & $3^{3}$ & $C 1$ & 0 & yes & 2,3 & 3 \\
2 & -30 & -63 & $-3^{5}$ & $3^{3}$ & $C 3$ & $-2^{15} \cdot 3 \cdot 5^{3}$ & yes & 1 & 4 \\
3 & 0 & 7 & $-3^{9}$ & $3^{3}$ & $C 6$ & 0 & yes & 1,4 & 1 \\
4 & -270 & 1708 & $-3^{11}$ & $3^{3}$ & $C 8$ & $-2^{15} \cdot 3 \cdot 5^{3}$ & & 3 & 2 \\
5 & 0 & 1 & $-3^{5}$ & $3^{5}$ & $C 1$ & 0 & & 7 & 7 \\
6 & 0 & -2 & $-3^{7}$ & $3^{5}$ & $C 3$ & 0 & yes & 8 & 8 \\
7 & 0 & -20 & $-3^{11}$ & $3^{5}$ & $C 6$ & 0 & yes & 5 & 5 \\
8 & 0 & 61 & $-3^{13}$ & $3^{5}$ & $C 8$ & 0 & & 6 & 6 \\
\hline
\end{tabular}




\section{REFERENCES}

[1] I. Miyawaki, Elliptic curves of prime power conductor with $Q$-rational points of finite order, Osaka J. Math., 10 (1973), 309-323.

[2] L. J. Mordell, Diophantine equations, Academic Press London and New York, (1969).

[3] A. Néron, Modèles minimaux des variétés abéliennes sur les corps locaux et globaux, Publ. Math. I.H.E.S. no. 21 (1965), 5-125.

[ 4 ] O. Neumann, Die elliptischen Kurven mit den Führern $3.2^{\mathrm{m}}$ und $9.2^{\mathrm{m}}$, Math. Nachr., 48 (1971), 387-389.

[ 5 ] A. P. Ogg, Abelian curves of 2-power conductor, Proc. Camb. Phil. Soc., 62 (1966), $143-148$.

[ 6 ] _ - Abelian curves of small conductor, J. reine angew. Math., 226 (1967), 205215.

[ 7 ] - Elliptic curves and wild ramification, Amer. J. Math., 89 (1967), 1-21.

[ 8 ] J. Vélu, Courbes elliptiques sur $Q$ ayant bonne réduction en dehors de $\{11\}, \mathrm{C} . \mathrm{R}$. Acad. Sci. Paris, 273 (1971), 73-75.

[ 9 ] — - Isogénies entre courbes elliptiques, C.R. Acad. Sci. Paris, 273 (1971), 238241.

[10] T. Hadano, Remarks on the Conductor of an Elliptic Curve, Proc. Jap. Acad., 48 (1972), 166-167.

Nagoya University 